\title{
A surface electromyographic examination of the serratus anterior during exercise, weight, and order-dependent variations of the bench press
}

Alex Caravan ${ }^{1}$, John O. Scheffey ${ }^{2}$, Kyle L. Rogers ${ }^{3}$, Kyle J. Boddy ${ }^{4}$, Michael E. O'Connell

${ }^{1,2,4}$ Driveline Baseball, Research \& Development, Kent, WA USA

${ }^{3}$ Driveline Baseball, High Performance, Kent, WA USA

Corresponding Authors:

Kyle L. Rogers, Kyle J. Boddy

19612 70th Avenue South, Unit 2-4

Kent, WA 98032

Email addresses: klrogers18@gmail.com, kyle@drivelinebaseball.com 


\begin{abstract}
The purpose of this study was to examine differences in the Electromyography (EMG) amplitude of the serratus anterior between the dumbbell bench press and barbell bench press, controlling for weight, order of sets, and dominance of arm. Forty (40) men aged $22.6 \pm 2.5$ years who were either college or professional baseball pitchers were block randomized into four exercise order-specific subgroups and then performed, depending on the aforementioned order-specific sections, 5-rep sets of both dumbbell and barbell bench press at both 95 and $135 \mathrm{lbs}$. Each pitcher was instructed in the proper usage of the exercise and assigned a standardized 90 -second rest period between the four sets. After receiving the raw EMG signal, proper band-pass filtering, rectifying, moving average-smoothing, and normalization of the EMG amplitude was commenced in order to extract meaningful EMG amplitude results. Statistically significant differences were assessed through a pair of non-parametric tests in the Mann-Wilcox and Wallis-Kruskal test, and the dumbbell bench press sets registered higher average mean frequencies at a $p$-value of 0.0008 . The subject's inclusion in one of the four sections registered at a similarly statistically significant $p$-value of 0.00008 .
\end{abstract}

\title{
Introduction
}

Proper function of the scapula and adequate shoulder strength are a primary concern for overhead athletes. Weakness and instability in overhead stabilizers is often a culprit for poor force generation and can leave athletes vulnerable to shoulder injury. The serratus anterior muscle is critical to both scapular upward rotation and scapula stabilization during overhead movements, and poor serratus anterior strength can be a culprit for poor overall scapula movement (Kibler, 1998). As part of an effort to improve maximal upper-body strength and stability in overhead athletes, bench press variations are commonly implemented in strength training, and upward rotators of the scapula like the serratus anterior are a targeted area for these exercises. It follows that bench press variations eliciting higher serratus anterior activation may be preferable for throwing athletes.

Several studies exist that examine muscle activation of large muscles in the upper extremity during bench press exercises. Bench press has been shown to be more effective for eliciting activation in the serratus anterior compared to push-ups and wall push-ups (Martins et al., 2008), but to our knowledge the effectiveness of different implementations of the bench press variations on training the serratus anterior has only been sparingly touched on. Anecdotally, dumbbell bench press is commonly believed to allow better scapular movement and more serratus anterior activation than barbell bench press, but there is little empirical data to support this claim.

This study involved measurements of electromyography (EMG) amplitude to examine differences across various exercises and weights, following similar precedent from past studies (Decker et al., 1999). EMG is a method used to detect and record electric potential across skeletal muscles as a response to muscle stimulation. However, EMG readings are subject to many forms of noise, including equipment calibration, background electromagnetic radiation, movement of electrodes, randomness of muscle firing patterns, and electrocardiography signal. Therefore, several analytical methods were necessary in 
order to separate the signal and the noise; filtering, smoothing, and normalization of the EMG signal were all employed (Caravan et al. 2018).

The purpose of this study was to use EMG sensors to examine serratus anterior activation in strength-trained males performing barbell and dumbbell bench press at two different weights and in four different orders, and to determine if there is a statistically significant difference in the EMG amplitude of the serratus anterior between the two bench press variations, weights, and orders. Weights of 95 pounds and 135 pounds were used for both barbell bench press and dumbbell bench press (Figs. 1 and 2).

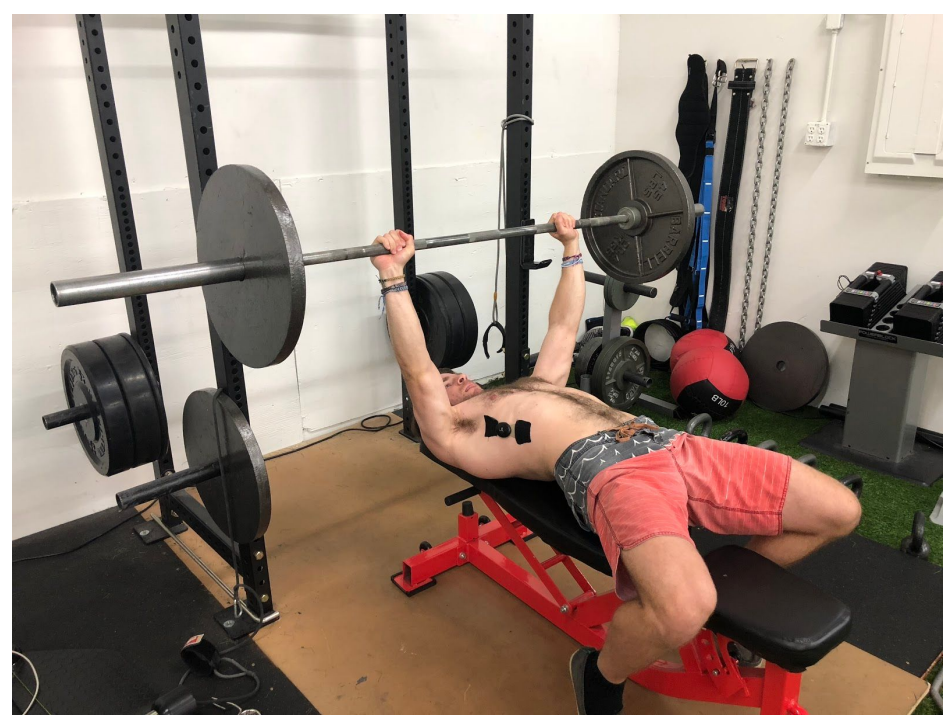

Figure 1 - an example of a subject performing barbell bench press with 135 pounds

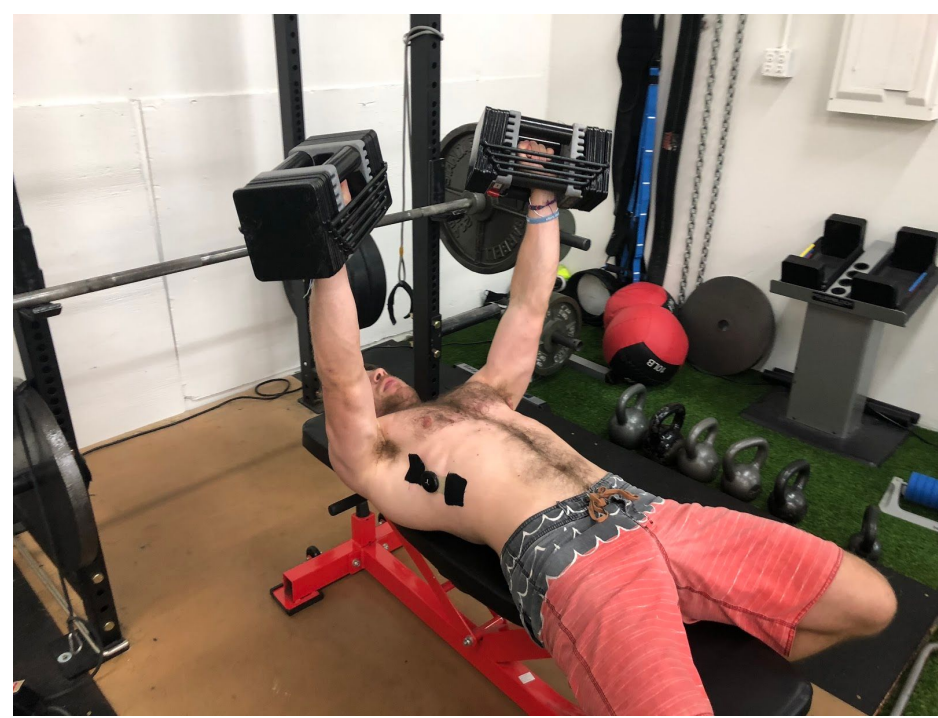

Figure 2 - an example of a subject performing dumbbell bench press with 67.5 pound dumbbells 
Bench press variations that result in higher serratus anterior EMG amplitude may indicate higher muscle activation and may be more beneficial for the strength training of overhead throwing athletes.

\section{Methods}

\section{Subjects}

The study was approved by Hummingbird IRB, who granted ethical approval to carry out the study (Hummingbird IRB\# 2018-17) at the author's facilities. Subjects were given a verbal explanation of the study by the investigators, and then given an Informed Consent form to review and sign. Forty healthy college and professional baseball players and employees at the Driveline Baseball facility in Kent, Washington were recruited for the study (age $=22.6 \pm 2.5$ years; height $=73.0 \pm 2.5$ inches; body weight $=206.3 \pm 17.2 \mathrm{lbs}$ ). Inclusion criteria requested subjects to be men between 18 and 40 years of age. Subjects were required to be able to lift at least 135 pounds from a supine position on a flat bench and to be pain and discomfort free in the upper extremities, specifically the forearm, elbow, shoulder, neck or any other area during the study. If pain or discomfort was experienced at any point during the trials, the study was stopped and the subject's data was discarded.

\section{Procedures}

Subjects were recruited to participate in the study on days when they were scheduled for a weight training session. Trials were completed after a warm-up and before a full session of weight training. Once ready to participate, subjects were selected from the general population and placed into four distinct sections of 10 subjects through a block randomization process. After cleaning the application sites, a Somaxis Cricket EMG sensor (Somaxis Inc., San Francisco, CA, USA) was applied vertically across a standardized site on the serratus anterior of both the left and right arms. The EMG sensors were held in place using kinesiology tape (RockTape, Inc., Campbell, CA, USA).

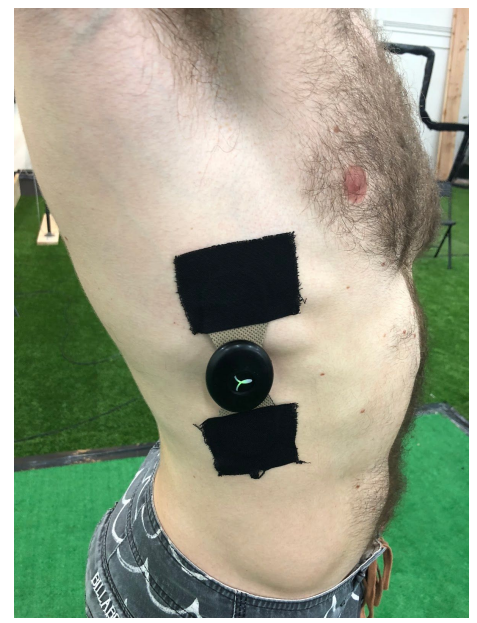

Figure 3 - application of the Somaxis EMG sensor to the right serratus anterior of the subject 


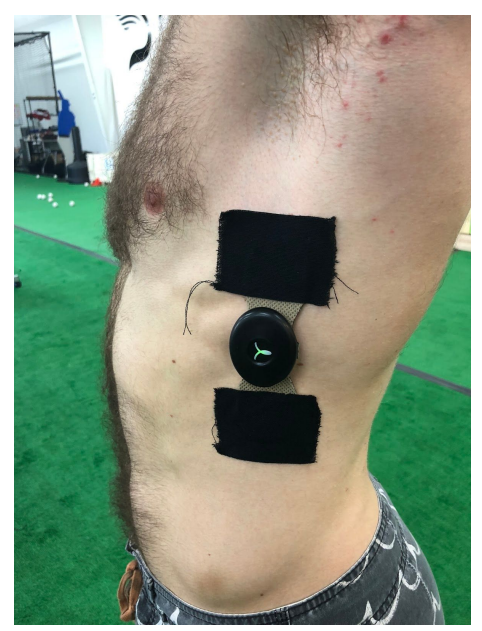

Figure 4 - application of the Somaxis EMG sensor to the left serratus anterior of the subject

Prior to performing the bench press exercises, maximum voluntary isometric contraction (MVIC) data for each subject was collected, to normalize future EMG readings for the bench press exercises. Subjects were first instructed to raise their right arm to $125^{\circ}$ of shoulder abduction in the scapular plane and a goniometer was used to measure the exact range of motion. Subjects were instructed, with their arm raised in the same measured position, to resist a downward force of 5 pounds applied to the humerus with a hand-held dynamometer (Lafayette Instrument Co., Lafayette, IN, USA) by an investigator, to achieve MVIC. The same MVIC procedure was then performed on the left arm. Manual resistance at a shoulder abduction angle of about $120^{\circ}$ has been shown to achieve higher serratus anterior activation than other MVIC positions (Jung \& Moon, 2015).

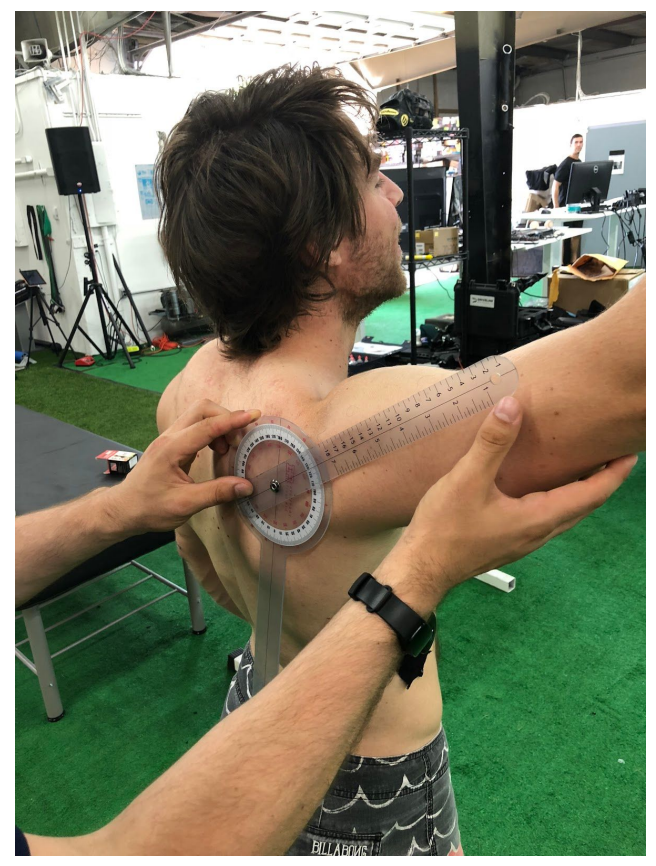

Figure 3 - measurement of the first MVIC position with goniometer 


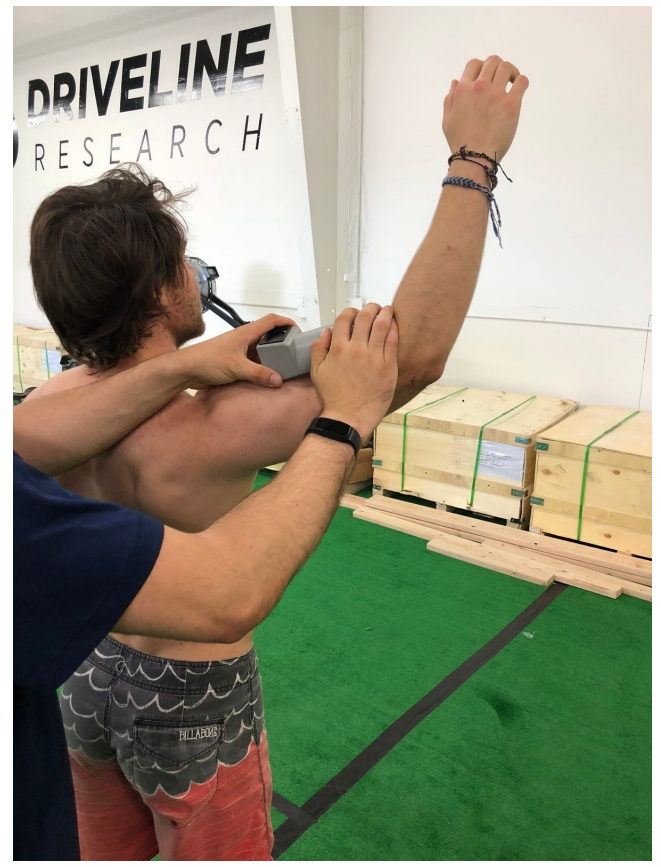

Figure 4 - an example of the first MVIC test done on subjects

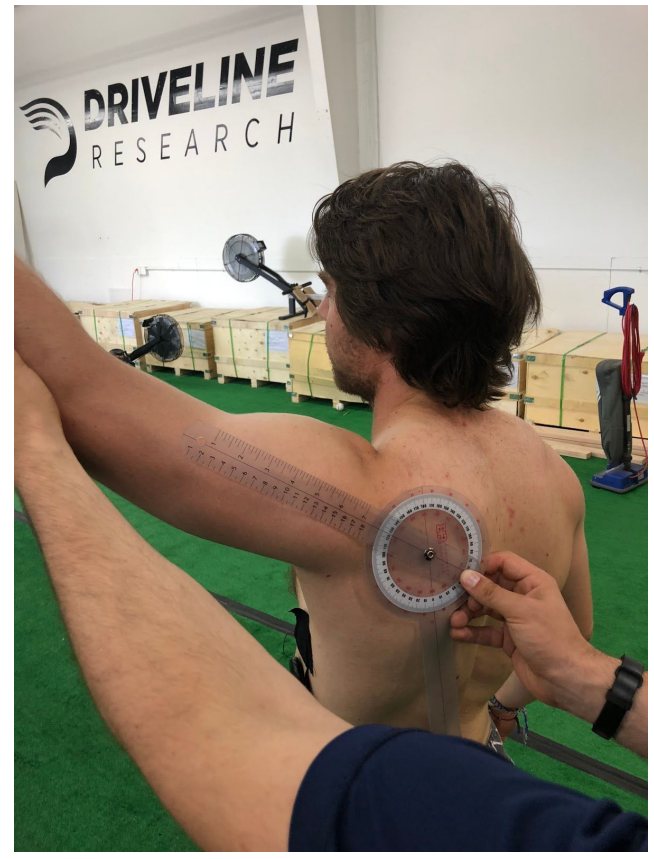

Figure 5 - measurement of the second MVIC position with a goniometer 


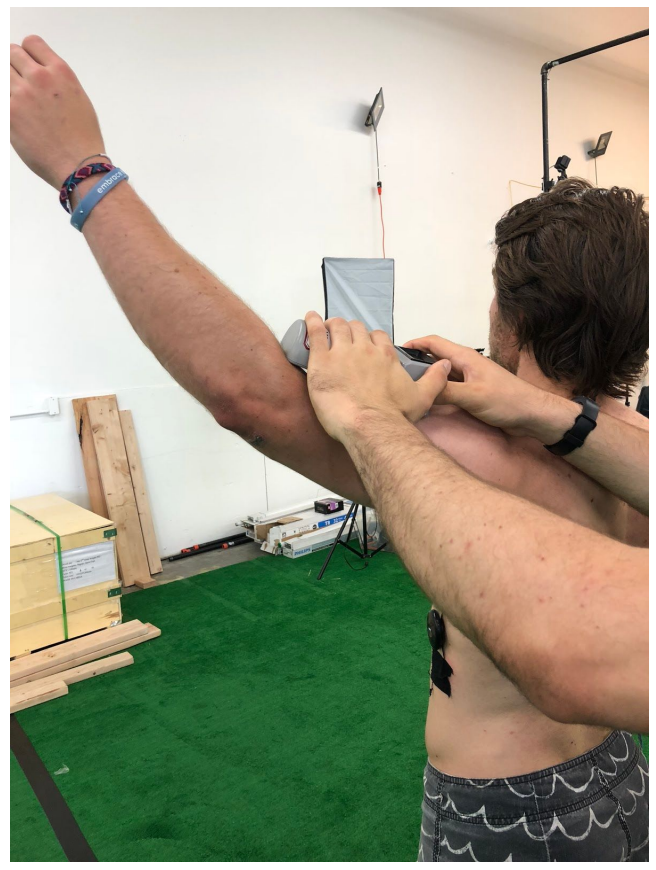

Figures 6 - an example of the second MVIC test done on subjects

Subjects were then given a brief explanation of the proper technique for bench press, and instructed to engage the latissimus dorsi muscles through the exercise. Subjects completed four sets of five reps of bench press, with a standardized $90 \mathrm{~s}$ rest period between each set. Subjects were told to perform the sets at a moderate pace and intensity. Four different weights were used in the trials, corresponding to the four sets: (Make this not a table / tabular data, just use bullet points)

- Light dumbells - two 47.5 pound dumbbells, totalling 95 pounds

- Light barbell - two 25 pound plates on a dumbbell, totalling 95 pounds

- Heavy dumbells - two 67.5 pound dumbbells, totalling 135 pounds

- Heavy barbell - two 45 pound plates on a dumbbell, totalling 135 pounds

The order of the lifts was varied for each of the four sections. The orders of the lifts performed are listed in Table 1 below.

Table 1

\begin{tabular}{|l|l|l|l|l|}
\hline Sections & Set 1 & Set 2 & Set 3 & Set $\mathbf{4}$ \\
\hline Section 1 & Light barbell & Light dumbbells & Heavy barbell & Heavy dumbbells \\
\hline Section 2 & Light dumbbells & Light barbell & Heavy dumbbells & Heavy barbell \\
\hline Section 3 & Light barbell & Light dumbbells & Heavy dumbbells & Heavy barbell \\
\hline Section 4 & Light dumbbells & Light barbell & Heavy barbell & Heavy dumbbells \\
\hline
\end{tabular}


The above weights were chosen as a moderate load for barbell and dumbbell bench press. The majority of the athletes in-gym are able to bench press weights well above 135 pounds. The 90 second rest period was chosen to provide adequate recovery for subjects between sets.

Raw EMG signals were collected at $1,000 \mathrm{~Hz}$ by a Somaxis Cricket EMG sensor (Somaxis Inc., San Francisco, CA, USA). Data was sent in real time to an iPad via Bluetooth and recorded by the Chirp for Cricket app (Somaxis Inc., San Francisco, CA, USA).

\section{Data Processing}

As referenced in multiple EMG-centered studies, multiple processes are required to derive tangible results from the EMG signal. Similar protocols were followed from past studies that used the identical equipment to reproduce a workable copy of the raw data (Caravan et al., 2018).

First, a band pass filter with a low end cut-off of $10 \mathrm{~Hz}$ and high end cut-off of $450 \mathrm{~Hz}$ was used (the lower range is typically used for removing electrical noise wire sway, biological artifacts and other sources of electrical noise while the higher range is banked on for erasing tissue noise at the site of the electrode placement) (De Luca et al., 2010). Only frequency components within that range were kept, and were then filtered with a Butterworth filter to produce a dataset with minimal passband ripple (Luca, 2003).

The output was then smoothed via a 200-window sized moving average, a popular choice in EMG analysis, to further separate the noise from the signal and have a better template for the identification of the four working sets, the MVIC signal, and the rest periods, as the electrodes were on the body of the subject during the entire continuous time period (Dieterich et al., 2017).

Once the MVIC positions were identified, the max MVIC value was extracted and used to normalize the data, a process used to enable appropriate comparisons between subjects (Boettcher et al., 2008). Normalization of the filtered signal is a common technique in EMG analysis (Halaki \& Ginn, 2012).

\section{Statistical Analysis}

Spectral analysis was performed on the filtered, smoothed, and normalized data, as the relative amplitude of the individual EMG waves' frequency content was extracted via a Fast Fourier Transform (Fele-Zorz et al. 2008). The mean and medium frequencies, along with the standard error of means and standard deviations of the sample points were taken in consideration during specific analyses of differences.

The differences in group means were analyzed through both a parametric and non-parametric lens. First a one-way ANOVA was used to determine the significance of differences in group means where there were more than two factor levels. However, not all of the underlying assumptions to using ANOVA methods were easily fulfilled. While the sample points were independently and randomly obtained from the population as a whole, the two other key assumptions for ANOVA tests-normality and homogeneity 
of variances-were not as readily fulfilled. Running the Shapiro Test for Normality on the residuals of most fitted ANOVA tests returned highly significant p-values (both at a 0.05 and 0.01 alpha level), while Leneve's Test for Homogeneity of Variance also returned similar results for most combinations of factors. A further investigation into the normality of the distributions of mean frequencies examined the kurtosis of both the order-dependent distributions (as in the subjects divided into their respective aforementioned Sections 1-4) as well as the data for each individual set, with most ranging outside an expected range of $-/+2$; the full figures are referenced in Table 2 below. To be fair, the kurtosis magnitudes indicated leptokurtosis rather than platykurtosis, with the latter commonly being the bigger cause for grief when it comes to Type I error (Cain et. al, 2017). Nevertheless, enough doubt was called to invoke non-parametric tests, so we employed both the Mann-Wilcox Tests and the Kruskal-Wallis Test as appropriate (when comparing means of just two groups and more than 2 groups, respectively).

Table 2

\begin{tabular}{|l|r|}
\hline Distribution & Kurtosis Values \\
\hline Set 1 Mean Freq & 2.1051 \\
\hline Set 2 Mean Freq & 5.4955 \\
\hline Set 3 Mean Freq & 9.6220 \\
\hline Set 4 Mean Freq & 0.1627 \\
\hline Section 1 Mean Freq & 2.1161 \\
\hline Section 2 Mean Freq & 2.1776 \\
\hline Section 3 Mean Freq & 4.5020 \\
\hline Section 4 Mean Freq & 8.5546 \\
\hline
\end{tabular}

\section{Results}

First, the descriptive statistics of the mean frequencies for the subject pool were initially pooled as followed, where both electrodes for each subject split out as their own data point and then sorted into the specific working set and ordered exercise section. The data is found in Table 3, below:

Table 3

\begin{tabular}{|c|l|l|l|l|l|l|l|l|l|}
\hline \multicolumn{2}{|c|}{$\mathbf{n}=\mathbf{8 0}$} & \multicolumn{2}{|c|}{ Set1 Averages } & \multicolumn{2}{c|}{ Set2 Averages } & \multicolumn{2}{c|}{ Set3 Averages } & \multicolumn{2}{c|}{ Set4 Averages } \\
\hline \multirow{2}{*}{ Groups } & Side & $\begin{array}{l}\text { Mean } \\
\text { Freq }\end{array}$ & $\begin{array}{l}\text { Mdn } \\
\text { Freq }\end{array}$ & $\begin{array}{l}\text { Mean } \\
\text { Freq }\end{array}$ & $\begin{array}{l}\text { Mdn } \\
\text { Freq }\end{array}$ & $\begin{array}{l}\text { Mean } \\
\text { Freq }\end{array}$ & $\begin{array}{l}\text { Mdn } \\
\text { Freq }\end{array}$ & $\begin{array}{l}\text { Mean } \\
\text { Freq }\end{array}$ & $\begin{array}{l}\text { Mdn } \\
\text { Freq }\end{array}$ \\
\hline
\end{tabular}




\begin{tabular}{|l|l|r|r|r|r|r|r|r|r|}
\hline Section 1 & & $\mathbf{8 . 7 2 1}$ & $\mathbf{0 . 1 1 2}$ & $\mathbf{1 0 . 0 8 3}$ & $\mathbf{0 . 1 4 3}$ & $\mathbf{9 . 4 1 8}$ & $\mathbf{0 . 0 8 7}$ & $\mathbf{8 . 7 4 5}$ & $\mathbf{0 . 1 4 5}$ \\
\hline & $L$ & 8.260 & 0.105 & 9.124 & 0.157 & 8.457 & 0.097 & 8.336 & 0.178 \\
\hline & $R$ & 9.182 & 0.120 & 11.041 & 0.129 & 10.380 & 0.077 & 9.153 & 0.112 \\
\hline & $L$ & 8.895 & 0.107 & 8.984 & 0.172 & 9.850 & 0.154 & 8.818 & 0.085 \\
\hline & $R$ & 8.723 & 0.143 & 9.199 & 0.207 & 10.308 & 0.198 & 8.681 & 0.095 \\
\hline Section 3 & & $\mathbf{1 1 . 3 4 5}$ & $\mathbf{0 . 2 1 0}$ & $\mathbf{9 . 4 9 9}$ & $\mathbf{0 . 1 5 1}$ & $\mathbf{9 . 3 1 5}$ & $\mathbf{0 . 1 6 4}$ & $\mathbf{9 . 5 9 4}$ & $\mathbf{0 . 2 0 3}$ \\
\hline & $L$ & 10.942 & 0.203 & 9.256 & 0.142 & 9.595 & 0.176 & 9.849 & 0.201 \\
\hline & $R$ & 11.749 & 0.218 & 9.742 & 0.160 & 9.036 & 0.153 & 9.339 & 0.206 \\
\hline Section 4 & & $\mathbf{1 5 . 0 6 5}$ & $\mathbf{0 . 2 4 9}$ & $\mathbf{1 1 . 8 3 7}$ & $\mathbf{0 . 1 2 2}$ & $\mathbf{1 0 . 2 6 5}$ & $\mathbf{0 . 1 7 7}$ & $\mathbf{9 . 7 2 8}$ & $\mathbf{0 . 1 1 2}$ \\
\hline & $L$ & 14.187 & 0.236 & 11.771 & 0.115 & 10.430 & 0.172 & 8.914 & 0.109 \\
\hline & $R$ & 15.943 & 0.262 & 11.903 & 0.129 & 10.099 & 0.182 & 10.541 & 0.115 \\
\hline & & $\mathbf{1 0 . 9 8 5}$ & $\mathbf{0 . 1 7 4}$ & $\mathbf{1 0 . 1 2 7}$ & $\mathbf{0 . 1 5 1}$ & $\mathbf{9 . 7 6 9}$ & $\mathbf{0 . 1 5 1}$ & $\mathbf{9 . 2 0 4}$ & $\mathbf{0 . 1 3 7}$ \\
\hline
\end{tabular}

We looked at comparing the group means through a number of factor-based group designations: a) the four individual sets; b) the four specific exercise-specific orders (coded in sections 1 through 4); c) the four different combinations of exercise and weight; $d$ ) the light (sets 1 and 2) versus heavy (sets 3 and 4) splits; and e) the dumbbell versus barbell splits for the whole population ( 80 total sets with the dumbbells versus the barbells).

Before delving further, here is another preliminary set of descriptive statistics, but now for the segmented weight and exercise-specific sections of the data we want to explore below in Table 4 . Barbell and dumbbell are going to be colloquially referred to by a shorthand initial here, and farther on in the paper.

Table 4

\begin{tabular}{|c|c|c|}
\hline Group & $\begin{array}{l}\text { Avg Mean } \\
\text { Freqs }\end{array}$ & $\begin{array}{l}\text { Avg Mdn } \\
\text { Freqs }\end{array}$ \\
\hline b light & 9.7165 & 0.1276 \\
\hline d light & 11.3960 & 0.1979 \\
\hline
\end{tabular}




\begin{tabular}{|l|r|r|}
\hline b heavy & 9.3026 & 0.1131 \\
\hline d heavy & 9.6706 & 0.1753 \\
\hline light total & 10.5563 & 0.1627 \\
\hline heavy total & 9.4866 & 0.1442 \\
\hline b total & 9.5096 & 0.1204 \\
\hline d total & 10.5333 & 0.1866 \\
\hline
\end{tabular}

Now, examining first the four-level factors among different groups (and subgroups of the data), we presented the ANOVA P-value alongside the more circumstantially sound Kruskal-Wallis results. We analyzed among the four-leveled factors covered above in a) through $\mathrm{c}$ ).

Looking at the multi-group (more than two groups) mean analysis, we found significant results for most scenarios, with the full results for both the Kruskal-Wallis and the ANOVA results included in Table 5. The exception seems to be that the specific set number was not significant amongst the whole subject population when looking at the mean frequencies.

Table 5

\begin{tabular}{|r|l|l|r|r|}
\hline $\mathbf{N}$ & Mean Freq & Factor (4 Levels) & \multicolumn{1}{l|}{ KW P-value } & \multicolumn{1}{l|}{ ANOVA P-value } \\
\hline 10 & Set 1 Mean Freq & Sections & $1.23 \mathrm{E}-06$ & $1.52 \mathrm{E}-05$ \\
\hline 10 & Set 2 Mean Freq & Sections & 0.0056 & 0.0072 \\
\hline 10 & Set 3 Mean Freq & Sections & 0.0020 & 0.4280 \\
\hline 10 & Set 4 Mean Freq & Sections & 0.0034 & 0.3720 \\
\hline 40 & All & Exercise \& Weight & 0.0041 & 0.0004 \\
\hline 40 & All & Sections & $7.59 \mathrm{E}-05$ & $3.06 \mathrm{E}-06$ \\
\hline 40 & All & Set \# & 0.2108 & 0.0077 \\
\hline
\end{tabular}

We then employed the Dunn Test to look at some of the more extreme differences for the group of mean frequencies as a whole. We examined both a) the combination of the Exercise \& Weight and b) the four singular section defining orders. Here are the following outputs from both factors, taken as screenshots from Rstudio. 
Figure 7: Dunn Test for Exercise \& Weight

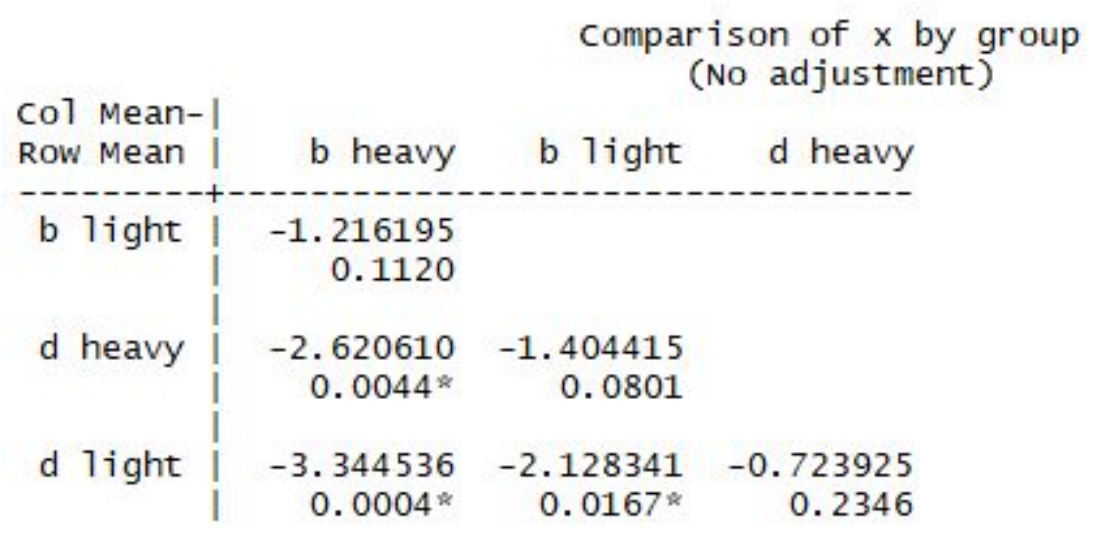

Figure 8: Dunn Test for Order

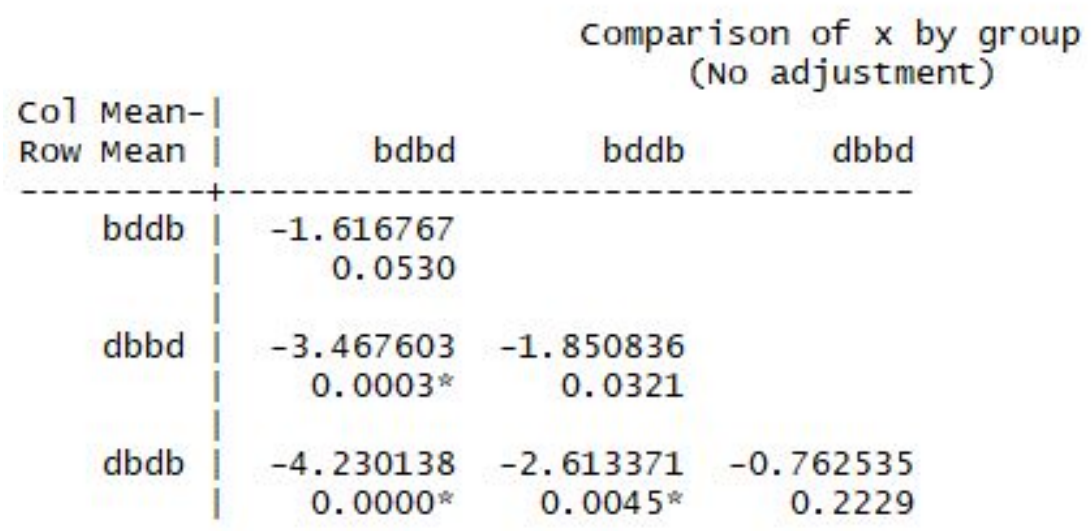

The results seem to indicate that the greater EMG activation is accomplished in the lighter sets and the dumbbell sets, with the peak differences appropriately being in the dumbbell light sets versus the barbell heavy ones. On top of that, Section 2 (which went light dumbbell, light barbell followed by heavy dumbbell, heavy barbell) showcased the greatest and most significant differences from its order-specific peers, with the greatest disparity coming when paired with Section 1 (barbell dumbbell barbell dumbbell).

Now, looking at only two-level factor comparisons, we relied on the similarly non-parametric Wilcox Test. Out of all the individual set subgroup frequencies we found the most significant results for Set 1 and Set 3, followed by Set 2, although interestingly enough there was a bigger significant difference, when controlling for exercise, between the heavy sets subgroup rather than the light sets (both were significant). The full table of information is below in Table 6 .

\section{Table 6}




\begin{tabular}{|r|l|l|l|}
\hline $\mathbf{N}$ & Mean Freq & Factor & P-value \\
\hline 10 & Set 1 Mean Freq & Exercise & $1.14 \mathrm{E}-05$ \\
\hline 10 & Set 2 Mean Freq & Exercise & 0.0402 \\
\hline 10 & Set 3 Mean Freq & Exercise & 0.0143 \\
\hline 10 & Set 4 Mean Freq & Exercise & 0.2110 \\
\hline 20 & Light Sets & Exercise & 0.0414 \\
\hline 20 & Heavy Sets & Exercise & 0.0065 \\
\hline 40 & All & Weight & 0.1706 \\
\hline 40 & All & Exercise & 0.0008 \\
\hline
\end{tabular}

Finally, given that we also have access to each individual electrode (both right and left) and the denotation of whether each subject was right handed or left handed, we were able to code each electrode specific output as pertaining to the dominant or non-dominant side. We split out these sides as their own respective data points (so now we have a sample size of 80 ) to examine whether there were more pronounced differences on either the dominant or non-dominant side under many of the same controlling factors we announced previously. The results are found in Table 7 below, where we specified which non-parametric test we used, in conjunction with the appropriate number of factors levels in consideration (either 2 or 4 ).

Table 7

\begin{tabular}{|r|l|l|l|r|}
\hline $\mathbf{N}$ & $\begin{array}{l}\text { Mean Freq } \\
\text { Samples }\end{array}$ & Factor & $\begin{array}{l}\text { Test } \\
\text { Used }\end{array}$ & P-Value \\
\hline 80 & All & Section & K-Wallis & 0.4895 \\
\hline 80 & All & Dom vs Non-Dom & Wilcox & 0.1386 \\
\hline 20 & Set 1 & Dom vs Non-Dom & Wilcox & 0.8072 \\
\hline
\end{tabular}




\begin{tabular}{|r|l|l|l|r|}
\hline 20 & Set 2 & Dom vs Non-Dom & Wilcox & 0.1206 \\
\hline 20 & Set 3 & Dom vs Non-Dom & Wilcox & 0.5886 \\
\hline 20 & Set 4 & Dom vs Non-Dom & Wilcox & 0.4521 \\
\hline 40 & Dom Side & Exercise \& Weight & K-Wallis & 1.52 E-05 \\
\hline 40 & Non-Dom Side & Exercise \& Weight & K-Wallis & 0.0072 \\
\hline 40 & Dom Side & Exercise & Wilcox & 0.0023 \\
\hline 40 & Non-Dom Side & Exercise & Wilcox & 0.0025 \\
\hline 40 & Dom Side & Weight & Wilcox & 0.1122 \\
\hline 40 & Non-Dom Side & Weight & Wilcox & 0.2956 \\
\hline
\end{tabular}

While the choice of dominance wasn't significant when looking at the whole set of mean frequencies, or for that matter any of the four individual sets, the dominant side had a (barely) more significant difference when examining the exercise selection, and registered a lower $p$-value when it came to weight as well although neither dominant nor non-dominant groups generated a $p$-value low enough to qualify as significant when it came to the latter designation.

\section{Discussion}

The main purpose of this investigation was to examine differences in the muscle engagement of the serratus anterior dependent on an exercise choice between dumbbell bench press and barbell bench press. Additional control factors that were examined were the weight used; the order in which the sets were performed; and the arm-dominance of the athlete in question.

These differences were examined across both the larger population as a whole, and subdivisions of the population including each individual set, the order-oriented four sections, the dominant and non-dominant groups and both the weight and exercise-dictated subgroups. As many of these distributions showed starkly non-normal distributions and heterogeneous variances, we relied primarily on the non-parametric Mann-Wilcox and Kruskal Willis tests to dictate statistical significance.

The variety of controlling factors used, as well as the various subgroups leaves an intimidating horde of results, many of them significant. To unpack a little: dumbbell sets returned a greater average mean frequency (10.5333 vs 9.5096$)$ at an extremely significant $p$-value of 0.0008 . In addition, the difference 
in exercise resulted in a statistically significant difference in the first three sets; the larger "light sets" and "heavy sets" groups as a whole; and in both the dominant and non-dominant side groups.

The lighter sets also averaged a higher mean frequency than their heavier counterparts at 10.5563 vs 9.4866, although in the population as a whole, this did not register a significant $p$ value at a 0.05 alpha level (0.1706). However, weight did serve to produce an even smaller p-value of 0.0004 when combined with the exercise choice to form four different factor levels. In addition, when performing a Dunn Test of Multiple Comparisons by rank sums, the largest difference occurred in the light dumbbell sets versus the heavy barbell sets, with the former showing, on average, a greater mean frequency of 3.35 .

In addition, the order of the exercises performed (i.e. Sections 1 through 4) were a statistically significant predictor when examined on the whole population, returning a p-value of 3.06E-06, and further being significant in Sets 1 and 2. Via results from the Dunn Test, the greatest mean difference occurred in the ' $d b d b$ ' section vs the 'bdbd' section (or Section 2 vs Section 1), with the former showing, on average, a greater mean frequency of 4.23 .

As mentioned in earlier sections, a number of disclaimers and processes have to be kept in mind when attempting to generate statistical significance from EMG data. EMG data contains a notorious amount of noise interspersed among its signal, especially when it comes to drawing inferences about individuals' muscle activation (Vigotsky et al., 2015). To minimize the amount of noise that trickles down to the end product, the experiment was designed with much thought towards the standardization and maintenance of the raw data: each subject followed the exact same rest periods in between sets and were given the same instructions and advice about minimizing any movement or muscle recruitment not directly related to the set in question. Post-data collection, the study adopted endorsed filtering, smoothing, normalizing, and frequency spectrum feature extraction methods (Oskaya).

There have been studies in the past dealing with the effectiveness of using the bench press as an exercise for serratus anterior EMG activation: a 2008 study examined that the upper trapezius to serratus anterior EMG amplitude ratio was higher during the bench press on a stable surface versus both wall push-ups and push ups across both stable and unstable surfaces (Martins et al, 2008). A 2011 study found that the serratus anterior had a statistically significant higher mean during the bench press as compared to the wall press (Tucci et al. 2008). A 2017 study found that the completion of pre-activation, either on stable or unstable surfaces, raised the EMG level during the subsequent work sets at $a<.01$ significance level (Pirauá et al. 2017) and a 2003 study found that there was a statistically significant power output increase in explosive bench press throws, post the completion of a heavy bench press work set (Baker, 2003).

While the serratus anterior specifically has not been examined in contrasting barbell vs dumbbell bench press, there have been studies done in a similar vein but with different muscles and different exercises. The medial deltoid has been shown to have significantly greater activation during the barbell bench press vs Smith machine bench press (Schick et al., 2010) while serratus anterior EMG activation has been previously analyzed across a variety of lower-resistance rehabilitation exercises, with results pointing 
out exercises such as the push-up plus and "dynamic hug" as the greatest recruiters of the serratus (Decker et. al, 1999).

Although quite a lot of considerations, from exercise selection to weight selection to order selection, were taken in account for the experiment design, there still exist frontiers for more exploration. For example, future studies could investigate a greater range of weights, as here only two standardized weights were used for both exercises: $95 \mathrm{lbs}$ and $135 \mathrm{lbs}$. This could shed light on building a better understanding of whether the current hypothesis about EMG activation being greater in the "lighter" 95 Ib sets hold over a multi-factor test. Alternatively, the weights could be based off an individualized allometric proportion rather than set denotations of 95 to $135 \mathrm{lbs}$, to better reproduce the ease or difficulty each subject has when handling said resistance training.

Another point of future interest to control for would be differences in trunk inclinations and hand spacings during the exercises, a set of variables that have already been explored for the bench press but for different muscles--specifically the EMG activation of five different shoulder muscles have been explored under four different trunk inclinations and two different hand spacings (Barnett et. al, 1995). Another potential variable to fold in would be the provision of internal cues during the performance of the exercise, as previous studies found that muscles like the triceps brachii had statistically significant differences in EMG activation, dependent on specific-muscle coaching instruction (Daniels and Cook, 2017).

\section{Conclusion}

Our findings indicate that there is a very statistically significant increase in EMG amplitude for the serratus anterior during the dumbbell bench press, as compared to the barbell bench press, a finding that holds up both during the lighter and heavier weights. The normalized EMG amplitude also shows statistically significant differences based on the codified order in which the exercise were performed, with the dumbbell-leading orders registering the highest mean frequencies. A multitude of potential noise-eliciting sources prevent these findings from firmly linking EMG amplitude and muscle activation,

but it would appear to indicate the serratus anterior is being activated more during the dumbbell bench press, and "light" dumbbell press at that. These findings allow greater education and insight into the potential programming of athletes targeting serratus anterior activation and/or general arm health during their resistance training.

\section{References}

Kibler WB. 1998. The Role of the Scapula in Athletic Shoulder Function. The American Journal of Sports Medicine26:325-337. DOI: 10.1177/03635465980260022801.

Martins J., Tucci HT., Andrade R., Araújo RC., Bevilaqua-Grossi D., Oliveira AS. 2008.

Electromyographic Amplitude Ratio of Serratus Anterior and Upper Trapezius Muscles During 
Modified Push-Ups and Bench Press Exercises: Journal of Strength and Conditioning Research22:477-484. DOI: 10.1519/JSC.0b013e3181660748.

Decker MJ., Hintermeister RA., Faber KJ., Hawkins RJ. 1999. Serratus Anterior Muscle Activity During Selected Rehabilitation Exercises. The American Journal of Sports Medicine27:784-791. DOI: $10.1177 / 03635465990270061601$.

Caravan A., Scheffey JO., Briend SJ., Boddy KJ. 2018. Surface electromyographic analysis of differential effects in kettlebell carries for the serratus anterior muscles. PeerJ 6:e5044. DOI: 10.7717/peeri.5044.

Jung D., Moon D. 2015. The effects of shoulder joint abduction angles on the muscle activity of the serratus anterior muscle and the upper trapezius muscle while vibrations are applied. Journal of Physical Therapy Science27:117-119. DOI: 10.1589/ipts.27.117.

De Luca CJ, Donald Gilmore L, Kuznetsov M, Roy SH. 2010. Filtering the surface EMG signal: movement artifact and baseline noise contamination. Journal of Biomechanics 43(8):1573-1579

Luca GD. 2003. Fundamental Concepts in EMG Signal Acquisition. :34.

Fele-Zorz G., Kavsek G., Novak-Antolic Z., Jager F. 2008. A comparison of various linear and non-linear signal processing techniques to separate uterine EMG records of term and pre-term delivery groups. Medical \& biological engineering \& computing 46:911-22. DOI: $10.1007 / \mathrm{s} 11517-008-0350-y$.

Boettcher CE., Ginn KA., Cathers I. 2008. Standard maximum isometric voluntary contraction tests for normalizing shoulder muscle EMG. Journal of Orthopaedic Research: Official Publication of the Orthopaedic Research Society 26:1591-1597. DOI: 10.1002/jor.20675.

Halaki M, Ginn K. 2012. Normalization of EMG signals: to normalize or not to normalize and what to normalize to? In: Naik GR, ed. Computational Intelligence in Electromyography Analysis-A 


\section{Perspective on Current Applications and Future Challenges. London: InTech Open Access}

Publisher. 175-194

Hopkins KD., Weeks DL. 1990. Tests for Normality and Measures of Skewness and Kurtosis: Their Place in Research Reporting. Educational and Psychological Measurement 50:717-29.

Cain MK., Zhang Z., Yuan K-H. 2017. Univariate and multivariate skewness and kurtosis for measuring nonnormality: Prevalence, influence and estimation. Behavior Research Methods 49:1716-1735. DOI: 10.3758/s13428-016-0814-1.

Vigotsky, A. D., Beardsley, C., Contreras, B., Steele, J., Ogborn, D., \& Phillips, S. M. (2015a). Greater electromyographic responses do not imply greater motor unit recruitment and 'hypertrophic potential' cannot be inferred. The Journal of Strength \& Conditioning Research.

Martins J., Tucci HT., Andrade R., Araújo RC., Bevilaqua-Grossi D., Oliveira AS. 2008.

Electromyographic amplitude ratio of serratus anterior and upper trapezius muscles during modified push-ups and bench press exercises. Journal of Strength and Conditioning Research 22:477-484. DOI: 10.1519/JSC.0b013e3181660748.

OZKAYA U. Frequency Analysis Of EMG Signals With Matlab Sptool. :6. https://doi.org/10.1519/JSC.0b013e3181660748.

Pirauá A., Barros Beltrão N., Ximenes Santos C., Pitangui A., Araújo R. 2017. Analysis of muscle activity during the bench press exercise performed with the pre-activation method on stable and unstable surfaces. Kinesiology 49.

Baker D. 2003. Acute Effect of Alternating Heavy and Light Resistances on Power Output During Upper-Body Complex Power Training. Journal of strength and conditioning research / National Strength \& Conditioning Association 17:493-7. DOI: 
Schick E., Coburn J., Brown L., A Judelson D., Khamoui A., Tran T., P Uribe B. 2010. A Comparison of Muscle Activation Between a Smith Machine and Free Weight Bench Press. Journal of strength and conditioning research / National Strength \& Conditioning Association 24:779-84. DOI: 10.1519/JSC.0b013e3181cc2237.

Barnett C., Kippers V., Turner P. 1995. Effects of Variations of the Bench Press Exercise on the EMG Activity of Five Shoulder Muscles. The Journal of Strength \& Conditioning Research 9:222.

Daniels RJ., Cook SB. 2017. Effect of instructions on EMG during the bench press in trained and untrained males. Human Movement Science 55:182-188. DOI: 10.1016/i.humov.2017.08.010. 